Geometry $8 \mathcal{T}$ Topology Monographs

Volume 4: Invariants of knots and 3-manifolds (Kyoto 2001)

Pages 103-117

\title{
Knot invariants derived from quandles and racks
}

\author{
SEIICHI KAMADA
}

\begin{abstract}
The homology and cohomology of quandles and racks are used in knot theory: given a finite quandle and a cocycle, we can construct a knot invariant. This is a quick introductory survey to the invariants of knots derived from quandles and racks.
\end{abstract}

AMS Classification 57M25, 57Q45; 55N99, 18G99

Keywords Quandles, racks, cocycle knot invariants, knot colorings.

\section{Introduction}

Many knot invariants can be calculated and interpretated via knot diagrams. For example, the (fundamental) quandle of a knot and the rack of a framed knot $[12,19]$ can be computed diagrammatically.

In 1942, Takasaki [31] introduced the notion of a $k e i$, that is an algebraic object consisting of a non-empty set and a binary operation on it. A kei acts on itself by multiplication from the right side (the right translations). The notion of a kei is an abstraction of the notion of the symmetric transformations. In particular, the right translations of a kei are involutory $((x * y) * y=x$ for any elements $x, y)$. When we drop this condition from the kei, we obtain the notion of a quandle, that was introduced by Joyce [19] in 1982. He associated a quandle to a knot, called the knot quandle and proved that the knot quandle is a complete invariant of a knot (up to weak equivalence). At the same time, Matveev [23] proved a similar result independently. He called the notion a distributive groupoid. Kauffman studied knot quandles and knot crystals in [20]. In 1988, Brieskorn [1] introduced the notion of an automorphic set and, in 1992, Fenn and Rourke [12] introduced the notion of a rack. Automorphic sets and racks are the same notion, but their actions are the left translations and the right translations, respectively. They removed the idempotency condition $(x * x=x$ for any element $x$ ) from the quandle. Fenn and Rourke also generalized the notion of the knot quandle to the rack of a framed knot or a knot diagram. 
Although knot quandles and racks are strong invariants, it is not easy to use them to distinguish knots by direct calculations. The situation is similar to the knot group. An easy method to use them in order to distinguish knots is to calculate the representations in a given finite quandle/rack. Fox's 3-colorings are representations of the knot quandle to the dihedral kei of order 3 and generalized Fox's colorings ( $n$-colorings) are representations to the dihedral kei of order $n[11,16,25]$.

In the 1990's and the early 2000's, the homology and cohomology theory for quandles and racks appeared $[4,6,13,14]$. In order to distinguish two quandles or racks, we may calculate and compare their homology groups. Such methods cannot distinguish a knot and its dual knot (the mirror image with the reversed orientation), since they have isomorphic knot quandles. Carter, Jelsovsky, Kamada, Langford, and Saito (CJKLS) [4] introduced an invariant of a knot derived from a quandle cocycle. Associated with a finite quandle and a cocycle, we can construct a knot invariant. The cohomology theory of a quandle introduced in [4] is related to the homology theory of a rack introdued by Fenn, Rourke and Sanderson (FRS) in $[13,14]$, cf $[6,21]$ or section 4 .

The invariant of CJKLS corresponds to the evaluation, by a fixed cocycle, of the fundamental class reprersented by a knot diagram in the homology group in the sense of FRS. The invariants due to CJKLS and FRS are not invariants that can be derived only from the knot quandle/rack. In fact, a trefoil knot and its mirror image have isomorphic quandles and the 2-twist spun trefoil and its orientation reversed 2-knot have isomorphic quandles. However they can be distinguished by the invariants.

This is a quick introductory survey on the invariants of knots derived from quandles and racks. The author thanks the organizers of the conference for giving him an oppotunity to give a talk. The talk was introductory and concentrated on the classical knot case according to the organizer's request. However this article includes some information on the 2 -knot case.

\section{Keis, quandles and racks}

A $k e i, X$, is a non-empty set with a binary operation $(a, b) \mapsto a * b$ satisfying the following axioms:

(K1) For any $a \in X, a * a=a$.

(K2) For any $a, b \in X,(a * b) * b=a$. 
(K3) For any $a, b, c \in X,(a * b) * c=(a * c) *(b * c)$.

A quandle, $X$, is a non-empty set with a binary operation $(a, b) \mapsto a * b$ satisfying the following conditions:

(Q1) For any $a \in X, a * a=a$.

(Q2) For any $a, b \in X$, there is a unique $c \in X$ such that $a=c * b$.

(Q3) For any $a, b, c \in X$, we have $(a * b) * c=(a * c) *(b * c)$.

The three axioms correspond respectively to the Reidemeister moves of type I, II, and III (see [12], [20], for example).

A rack, $X$, is a non-empty set with a binary operation $(a, b) \mapsto a * b$ satisfying the following conditions:

(R1) For any $a, b \in X$, there is a unique $c \in X$ such that $a=c * b$.

(R2) For any $a, b, c \in X$, we have $(a * b) * c=(a * c) *(b * c)$.

By definition, a kei is a quandle and a quandle is a rack:

$$
\{\text { keis }\} \subset\{\text { quandles }\} \subset\{\text { racks }\} .
$$

Here are some typical examples.

- Any set $X$ with the operation $x * y=x$ for any $x, y \in X$ is a kei, which is called the trivial kei, the trivial quandle or the trivial rack.

- Let $n$ be a positive integer. For elements $i, j \in\{0,1, \ldots, n-1\}$, define $i * j \equiv 2 j-i \quad(\bmod n)$. Then $*$ defines a kei structure called the dihedral $k e i, R_{n}$. This set can be identified with the set of reflections of a regular $n$-gon with conjugation as the kei operation.

- A group $X=G$ with $n$-fold conjugation as the quandle operation: $a * b=$ $b^{-n} a b^{n}$.

- A $\Lambda\left(=\mathbf{Z}\left[T, T^{-1}\right]\right)$-module $M$ is a quandle with $a * b=T a+(1-T) b, a, b \in$ $M$, called an Alexander quandle. For example, $R_{4} \cong \mathbf{Z}_{2}\left[T, T^{-1}\right] /\left(T^{2}+1\right)$.

See $[1,12,19,23]$ for further examples.

A subset $S$ of a rack $X$ is called a generating set if any element of $X$ is obtained from the elements of $S$ by applying the operation suitably. If $X$ is a kei, we have

$$
\begin{aligned}
& x *(y * z)=((x *(y * z)) * z) * z \quad(\text { by } \quad \mathrm{K} 2) \\
& =((x * z) *((y * z) * z)) * z \quad(\text { by } \quad \mathrm{K} 3) \\
& =((x * z) * y) * z \quad(\text { by } \quad \mathrm{K} 2)
\end{aligned}
$$


for any $x, y, z$ of $X$. Using this, Takasaki [31] and Winker [32] (cf. [20], p. 195) proved that any element of a kei $X$ which is expressed by elements of $S$ can be re-expressed by using the same elements in the form as

$$
\left(\cdots\left(\left(\left(x_{1} * x_{2}\right) * x_{3}\right) * x_{4}\right) \cdots\right) .
$$

Such an expression is denoted by $x_{1} x_{2} x_{3} x_{4} \cdots$ or $x_{1}^{x_{2} x_{3} x_{4} \cdots}$ following [31] and [12]. For example,

$$
\begin{aligned}
(a *(b * c)) *(d * e) & =(((a * c) * b) * c) *(d * e) \\
& =(((((a * c) * b) * c) * e) * d) * e \\
& =a^{c b c e d e}
\end{aligned}
$$

Fenn and Rourke [12] introduced this notation for any rack $X$. They denote by $a^{b}$ the element $a * b$ of $X$ and assume that $a^{b^{c}}$ stands for $a^{\left(b^{c}\right)}=a *(b * c)$ and $a^{b c}$ stands for $\left(a^{b}\right)^{c}=(a * b) * c$. Then the axioms are stated as follows:

(R1) For any $a, b \in X$, there is a unique $c \in X$ such that $a=c^{b}$.

(R2) For any $a, b, c \in X$, we have

$$
a^{b c}=a^{c b^{c}} .
$$

The axiom R1 implies that the function $S_{x}: X \rightarrow X$ (acting from the right side) defined by $(u) S_{x}=u * x=u^{x}$ is a bijection. The axiom $\mathrm{R} 2$ implies that it is a homomorphism; $(u * v) S_{x}=(u) S_{x} *(v) S_{x}$ or $(u * v)^{x}=\left(u^{x}\right) *\left(v^{x}\right)$. We call it the right translation by the element $x$. For a word $W=x_{1}^{\epsilon_{1}} \cdots x_{k}^{\epsilon_{k}}$ consisting of elements of $X$ and for an element $u \in X$, we denote by $u^{W}$ the element (u) $S_{x_{1}}^{\epsilon_{1}} \cdots S_{x_{k}}^{\epsilon_{k}}$. In particular, $a^{b^{-1}}$ stands for the element $c$ with $c^{b}=a$. Then

$$
x^{y^{z}}=x^{z^{-1} y z}
$$

for any $x, y, z$ of $X$. Using this, we see that if $S$ is a generating set of a rack, then any element of the rack is expressed by $u^{W}$ for some $u \in S$ and a word $W$ consisting of elements of $S$. Refer to [12] for the details.

\section{Knot quandles and colored knot diagrams}

The quandle $Q(\mathcal{K})$ of a knot $\mathcal{K}$ and the rack $R(\mathcal{K})$ of a framed knot $\mathcal{K}$ were introduced in [19] and [12]. They can be calculated from a diagram.

Let $D$ be a diagram in $\mathbf{R}^{2}$ of an oriented knot $\mathcal{K}$, and let $E=\left\{x_{1}, \ldots, x_{m}\right\}$ be the set of the arcs of the diagram. We give each $\operatorname{arc}$ of the diagram $D$ a specific normal direction (co-orientation) determined by use of the orientations of $\mathcal{K}$ 
and $\mathbf{R}^{2}$. For a crossing point $\tau$, let $x_{i}, x_{j}$ and $x_{k}$ be the three arcs around $\tau$ such that $x_{k}$ is the over-arc at the crossing and $x_{i}$ is one of the under-arcs away from which the normal direction of $x_{k}$ points. We consider a relation

$$
x_{j}=x_{i} * x_{k}
$$

and call it the crossing relation at $\tau$ (see Fig. 1).

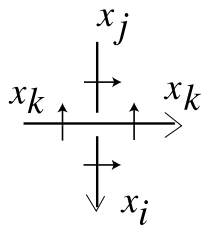

(1)

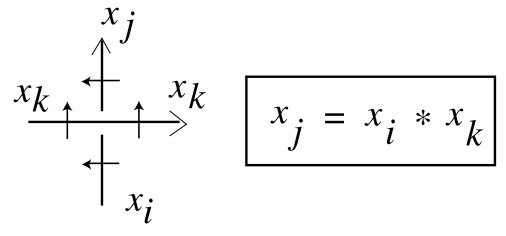

(2)

Figure 1

The kei $K(D)$, the quandle $Q(D)$, or the rack $R(D)$ of the diagram is defined to be the kei, the quandle or the rack that is generated by the set $E=\left\{x_{1}, \ldots, x_{m}\right\}$ and the defining relations are the crossing relations of the crossings of $D$.

The kei $K(D)$ can be defined even if the knot $\mathcal{K}$ is unoriented, since $x_{j}=x_{i} * x_{k}$ and $x_{i}=x_{j} * x_{k}$ are equivalent by the second axiom, $\mathrm{K} 2$.

By using Reidemeister moves, we see that $K(D)$ and $Q(D)$ are invariants of the knot $\mathcal{K}$. In fact, the quandle $Q(D)$ is isomorphic to the knot quandle $Q(\mathcal{K})$ of the knot $\mathcal{K}$, see $[12,19]$. Similarly, $R(D)$ is preserved by Reidemeister moves of type 2 and type 3 , and hence it is an invariant of the framed knot $\mathcal{K}$, where we assume the framing is the blackboard framing of the diagram. This is isomorphic to the rack $R(\mathcal{K})$ of the framed knot $\mathcal{K}$, see [12].

In general it is difficult to distinguish the isomorphism types of two given presentations of keis, quandles or racks. A convenient method is to use representations to a finite kei, quandle or rack $X$.

Let $\rho: K(D) \rightarrow X$ be a homomorphism. We call it a coloring of $D$ by $X$. We color the arc $x_{i}$ of the diagram $D$ with the element $\rho\left(x_{i}\right)$ of $X$. The crossing relation $x_{j}=x_{i} * x_{k}$ implies $\rho\left(x_{j}\right)=\rho\left(x_{i}\right) * \rho\left(x_{k}\right)$ in $X$. This is called the crossing relation for the coloring or the coloring condition.

For example, consider the case that $X$ is the dihedral kei $R_{3}=\{0,1,2\}$ of order three. The crossing relation implies that the three colors $\rho\left(x_{i}\right), \rho\left(x_{j}\right)$ and $\rho\left(x_{k}\right)$ are the same or all of them are distinct. This is the 3-coloring condition due to Fox, cf. [11, 25]. (In Exercise 6 of Chapter VI of [11], there is an additional 
condition that all three colors are actually used. The 3-coloring condition together this condition is also referred to as Fox's 3-coloring condition. In this paper, we do not assume it.)

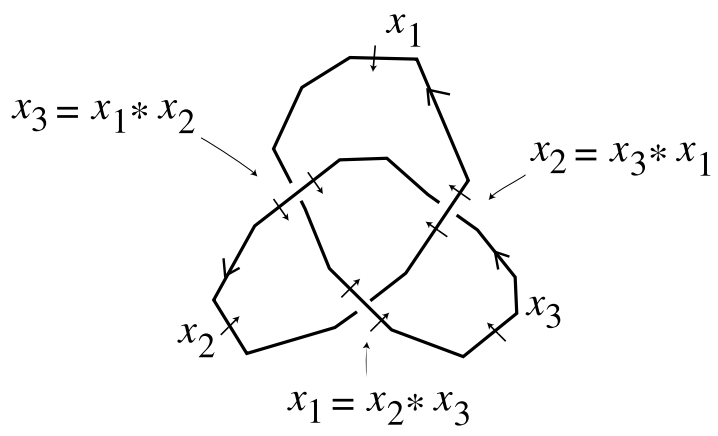

Figure 2

A typical diagram of the trefoil knot yields the presentation

$$
\left\langle x_{1}, x_{2}, x_{3} \mid x_{3}=x_{1} * x_{2}, \quad x_{1}=x_{2} * x_{3}, \quad x_{2}=x_{3} * x_{1}\right\rangle
$$

(see Fig. 2). Then the diagram has 9 Fox's 3-colorings. Three of them are trivial colorings and the other six are non-trivial colorings. Since the obvious diagram of the trivial knot has trivial colorings only, we see that the trefoil knot is not the trivial knot.

When we use the dihedral kei $R_{n}$ of order $n$, we have Fox's $n$-coloring. Fox's $n$-coloring is studied in [25]. In general, by use of a finite kei, we can consider colorings for unoriented knot diagrams. When we use a finite quandle or rack $X$ which is not involutory, diagrams must be oriented. A generalized $n$-coloring, called an $(n, r)$-coloring, was introduced by Silver and Williams [30]. It is interpretated as a coloring by a certain quandle $X$.

The knot group (the fundamental group of the knot complement) $G(\mathcal{K})$ can be calculated as the group $G(D)$ of a diagram $D$ that is the group with the generating set $E=\left\{x_{1}, \ldots, x_{m}\right\}$ and the Wirtinger relations $x_{j}=x_{k}^{-1} x_{i} x_{k}$ derived from the crossing points. The presentation of $G(D)$ is obtained from the presentation of $Q(D)$ by assuming $x_{j}=x_{i} * x_{k}$ to be $x_{j}=x_{k}^{-1} x_{i} x_{k}$. In fact, the knot group is a consequence of the knot quandle (see below or [12]).

For a quandle/rack $X$, assuming $x * y$ to be $y^{-1} x y$, we obtain a group. It is called the associated group of $X$ and denoted by $\operatorname{As}(X)$. More precisely $\operatorname{As}(X)$ is the group $F(X) / N$ where $F(X)$ is the free group generated by the elements of $X$ and $N$ is the normal subgroup of $F(X)$ generated by the words $(x * y) y^{-1} x y$ 
for $x, y \in X$, see [12]. A (group) presentation of $\operatorname{As}(X)$ is obtained from a (quandle/rack) presentation of $X$ by reading $x * y$ as $y^{-1} x y$, cf. Lemma 4.3 of [12]. Conversely, for a group $G$, define a binary operation $x * y$ by $y^{-1} x y$ and obtain a quandle. Such a quandle is called the conjugation quandle of $G$ and denoted by $\operatorname{conj}(G)$.

Joyce [19] and Matveev [23] proved the following theorem, which shows that the knot quandle is truely stronger than the knot group for some composite knots.

Theorem 3.1 (Joyce [19], Matveev [23]) Knots $\mathcal{K}$ and $\mathcal{K}^{\prime}$ with $Q(\mathcal{K}) \cong Q\left(\mathcal{K}^{\prime}\right)$ are weakly equivalent; namely, $\left(\mathbf{R}^{3}, \mathcal{K}\right)$ is homeomorphic to $\left(\mathbf{R}^{3}, \mathcal{K}^{\prime}\right)$ when we ignore the orientations of $\mathbf{R}^{3}$ and the knots.

For an oriented knot $\mathcal{K}$, let $-\mathcal{K}$ denote the knot with the reversed orientation and let $\mathcal{K}^{*}$ denote the mirror image. Then $Q(\mathcal{K}) \cong Q\left(-\mathcal{K}^{*}\right)$. If the knot $\mathcal{K}$ is invertible (i.e., $\mathcal{K} \cong-\mathcal{K}$ ), or amphicheiral (i.e., $\mathcal{K} \cong \mathcal{K}^{*}$ ), then $Q(\mathcal{K}), Q(-\mathcal{K})$, $Q\left(\mathcal{K}^{*}\right)$ and $Q\left(-\mathcal{K}^{*}\right)$ are isomorphic to each other. Therefore any invariant which is derived only from the quandle $Q(\mathcal{K})$ cannot distinguish the trefoil and its mirror image, since the trefoil is invertible. The invariants due to CJKLS and FRS can distinguish this pair and hence they are not invariants that are derived only from the knot quanadle.

\section{Homologies and cohomologies of a quandle and a rack}

For a rack $X$, let $C_{n}^{\mathrm{R}}(X)$ be the free abelian group generated by $n$-tuples $\left(x_{1}, \ldots, x_{n}\right)$ of elements of $X$ when $n$ is a positive integer and put $C_{n}^{\mathrm{R}}(X)=0$. Define a homomorphism $\partial_{n}: C_{n}^{\mathrm{R}}(X) \rightarrow C_{n-1}^{\mathrm{R}}(X)$ by

$$
\begin{aligned}
\partial_{n}\left(x_{1}, x_{2}, \ldots, x_{n}\right)=\sum_{i=1}^{n} & (-1)^{i}\left[\left(x_{1}, x_{2}, \ldots, x_{i-1}, x_{i+1}, \ldots, x_{n}\right)\right. \\
& \left.-\left(x_{1}^{x_{i}}, x_{2}^{x_{i}}, \ldots, x_{i-1}^{x_{i}}, x_{i+1}, \ldots, x_{n}\right)\right]
\end{aligned}
$$

for $n \geq 2$ and $\partial_{n}=0$ for $n \leq 1$. Then $C_{*}^{\mathrm{R}}(X)=\left\{C_{n}^{\mathrm{R}}(X), \partial_{n}\right\}$ is a chain complex. Let $C_{n}^{\mathrm{D}}(X)$ be the subset of $C_{n}^{\mathrm{R}}(X)$ generated by $n$-tuples $\left(x_{1}, \ldots, x_{n}\right)$ with $x_{i}=x_{i+1}$ for some $i \in\{1, \ldots, n-1\}$ if $n \geq 2$; otherwise let $C_{n}^{\mathrm{D}}(X)=0$. If $X$ is a quandle, then $\partial_{n}\left(C_{n}^{\mathrm{D}}(X)\right) \subset C_{n-1}^{\mathrm{D}}(X)$ and $C_{*}^{\mathrm{D}}(X)=\left\{C_{n}^{\mathrm{D}}(X), \partial_{n}\right\}$ 
is a sub-complex of $C_{*}^{\mathrm{R}}(X)$. In this case, let $C_{*}^{\mathrm{Q}}(X)$ be the quotient complex $C_{*}^{\mathrm{R}}(X) / C_{*}^{\mathrm{D}}(X)$. Let $G$ be an abelian group and let $\mathrm{W}$ stand for $\mathrm{R}, \mathrm{D}$ or $\mathrm{Q}$. Then we consider the homology and cohomology groups

$$
H_{n}^{\mathrm{W}}(X ; G)=H_{n}\left(C_{*}^{\mathrm{W}}(X) \otimes G\right), \quad H_{\mathrm{W}}^{n}(X ; G)=H^{n}\left(\operatorname{Hom}\left(C_{*}^{\mathrm{W}}(X), G\right)\right) .
$$

Definition 4.1 The $n$th rack homology and rack cohomology groups [13] of a rack/quandle $X$ with coefficient group $G$ are $H_{n}^{\mathrm{R}}(X ; G)$ and $H_{\mathrm{R}}^{n}(X ; G)$. The $n$th degeneration homology and degeneration cohomology groups of a quandle $X$ with coefficient group $G$ are $H_{n}^{\mathrm{D}}(X ; G)$ and $H_{\mathrm{D}}^{n}(X ; G)$. The $n$th quandle homology and quandle cohomology groups [4] of a quandle $X$ with coefficient group $G$ are $H_{n}^{\mathrm{Q}}(X ; G)$ and $H_{\mathrm{Q}}^{n}(X ; G)$.

The $n$th cocycle group of the cochain complex $\operatorname{Hom}\left(C_{*}^{\mathrm{Q}}(X), G\right)$ is called the quandle $n$-cocycle group and denoted by $Z_{n}^{\mathrm{Q}}(X ; G)$. We will omit the coefficient group $G$ as usual if $G=\mathbf{Z}$. Refer to $[13,14,15,17]$ for some calculations and applications of the rack homology groups, and $[4,5,6,7,21,24]$ for the quandle homology groups.

Theorem 4.2 (Universal Coefficient Theorem [6]) There exist split exact sequences

$$
\begin{array}{r}
0 \rightarrow H_{n}^{\mathrm{W}}(X) \otimes G \rightarrow H_{n}^{\mathrm{W}}(X ; G) \rightarrow \operatorname{Tor}\left(H_{n-1}^{\mathrm{W}}(X), G\right) \rightarrow 0 \\
0 \rightarrow \operatorname{Ext}\left(H_{n-1}^{\mathrm{W}}(X), G\right) \rightarrow H_{\mathrm{W}}^{n}(X ; G) \rightarrow \operatorname{Hom}\left(H_{n}^{\mathrm{W}}(X), G\right) \rightarrow 0 .
\end{array}
$$

By definition, there is a natural short exact sequence

$$
0 \rightarrow C_{*}^{\mathrm{D}}(X) \rightarrow C_{*}^{\mathrm{R}}(X) \rightarrow C_{*}^{\mathrm{Q}}(X) \rightarrow 0,
$$

which is split (in the weak sense), namely, for each $n$, the short exact sequence

$$
0 \rightarrow C_{n}^{\mathrm{D}}(X) \rightarrow C_{n}^{\mathrm{R}}(X) \rightarrow C_{n}^{\mathrm{Q}}(X) \rightarrow 0
$$

is split. Therefore we have a long exact sequence

$$
\cdots \stackrel{\partial_{*}}{\rightarrow} H_{n}^{\mathrm{D}}(X ; G) \rightarrow H_{n}^{\mathrm{R}}(X ; G) \rightarrow H_{n}^{\mathrm{Q}}(X ; G) \stackrel{\partial_{*}}{\rightarrow} H_{n-1}^{\mathrm{D}}(X ; G) \rightarrow \cdots .
$$

In [6], it was proved that this sequence is split into short exact sequences for small $n$ 's and it was conjectured that so is for all $n$ 's. This was proved by Litherland and Nelson [21].

Theorem 4.3 (Litherland and Nelson [21]) The short exact sequence (11) is split in the strong sense, that is, there exist splitting homomorphisms compatible with the boundary maps. Thus, for each $n$, there is a short exact sequence

$$
0 \rightarrow H_{n}^{\mathrm{D}}(X ; G) \rightarrow H_{n}^{\mathrm{R}}(X ; G) \rightarrow H_{n}^{\mathrm{Q}}(X ; G) \rightarrow 0 .
$$


Suppose that $X$ is a quandle with finitely many orbits, where orbits means orbits by the inner-automorphisms of $X$, i.e., orbits by the right action of $X$ on itself by the right translations. (In [19] orbits by all the automorphisms of $X$ are studied.) Let $\mathbf{Z}[\operatorname{Orb}(X)]$ be the free abelian group generated by the orbit set, $\operatorname{Orb}(X)$, of $X$. In $[6,17]$ it is shown that

$$
H_{1}^{\mathrm{D}}(X)=0, \quad H_{1}^{\mathrm{R}}(X) \cong H_{1}^{\mathrm{Q}}(X) \cong \mathbf{Z}[\mathrm{Orb}(X)], \quad \text { and } \quad H_{2}^{\mathrm{D}}(X) \cong \mathbf{Z}[\operatorname{Orb}(X)] .
$$

Hence

$$
H_{2}^{\mathrm{R}}(X) \cong H_{2}^{\mathrm{Q}}(X) \oplus \mathbf{Z}[\operatorname{Orb}(X)]
$$

In $[21]$ (Theorem 2.2) it is proved that

$$
H_{3}^{\mathrm{R}}(X) \cong H_{3}^{\mathrm{Q}}(X) \oplus H_{2}^{\mathrm{Q}}(X) \oplus \mathbf{Z}[\mathrm{Orb}(X) \times \operatorname{Orb}(X)] .
$$

The quandle (co)homology groups of some finite quandles were caluculated in [5] (Table 1). However there are errata due to the authors. They thank R. A. Litherland, S. Nelson [21] and T. Mochizuki [24] for pointing out and correcting them. Further calculations on homology/cohomology groups of finite Alexander quandles are found in their papers [21] and [24].

\section{$5 \quad$ Knot invariants}

Let $X$ be a finite quandle, let $G$ be an abelian group (written in multiplicative notation), and let $\phi \in C_{\mathrm{Q}}^{2}(X ; G)$ be a quandle 2-cocycle.

Let $D$ be an oriented knot diagram. Recall that a homomorphism $\rho: Q(D) \rightarrow$ $X$ is called a coloring and satisfies the crossing condition $\rho\left(x_{j}\right)=\rho\left(x_{i}\right) * \rho\left(x_{k}\right)$ at each crossing, where $x_{i}, x_{j}$ and $x_{k}$ are as before (see Fig. 1). For each crossing point $\tau$, we consider an element $W_{\phi}(\tau, \rho)$ of $G$ determined by

$$
W_{\phi}(\tau, \rho)=\phi\left(\rho\left(x_{i}\right), \rho\left(x_{k}\right)\right)^{\epsilon},
$$

where $x_{i}$ and $x_{k}$ are the arcs around $\tau$ corresponding the crossing relation $x_{j}=x_{i} * x_{k}$, and $\epsilon$ is the sign of the crossing point $\tau$. Consider the element

$$
\Phi_{\phi}(D)=\sum_{\rho} \prod_{\tau} W_{\phi}(\tau, \rho)
$$

of the group ring $\mathbf{Z} G$, where $\rho$ runs all colorings of $D$ by $X$ and $\tau$ runs all crossing points of $D$. Since $\phi$ is a cocycle, the value $\Phi_{\phi}(D)$ is preserved by Reidemeister moves. Therefore it is an invariant of the knot $\mathcal{K}$ represented by the diagram. Thus we denote this value by $\Phi_{\phi}(\mathcal{K})$ and call it the statesum invariant or the quandle cocycle invariant, [4]. If $\phi$ and $\phi^{\prime}$ are cocycles 
that are cohomologous then $\Phi_{\phi}(\mathcal{K})=\Phi_{\phi^{\prime}}(\mathcal{K})$. Thus this invariant depends on the quandle cohomology class of $\phi$. When $\phi$ is a trivial cocycle, the element $W_{\phi}(\tau, \rho)$ is the trivial element of $G$. In this case the invariant $\Phi_{\phi}(\mathcal{K})$ is equal to the number of all colorings of $D$ by $X$. In [8,2], the abelian extension $E=$ $E(X, G, \phi)$ is defined as $G \times X$ as a set, with the quandle operation $\left(g_{1}, x_{1}\right) *$ $\left(g_{2}, x_{2}\right)=\left(g_{1} \phi\left(x_{1}, x_{2}\right), x_{1} * x_{2}\right)$. The state-sum $\Phi_{\phi}(D)$ is an obstruction to extending the colorings of $D$ by $X$ to colorings by $E(X, G, \phi)$, see Theorems 4.1 and 4.5 of $[2]$.

Let $X$ be a finite quandle, let $G$ be an abelian group (written in multiplicative notation), and let $\theta \in C_{\mathrm{Q}}^{3}(X ; G)$ be a quandle 3 -cocycle.

A shadow coloring (or face coloring) of $D$ by $X$ is a function $\tilde{\rho}: \tilde{E} \rightarrow X$, where $\tilde{E}$ is the set of arcs of the diagram $D$ and the regions separated by the underlying immersed curve of the diagram, satisfying the coloring condition $\left(\tilde{\rho}\left(x_{j}\right)=\tilde{\rho}\left(x_{i}\right) * \tilde{\rho}\left(x_{k}\right)\right)$ and the condition that if $y_{i}$ and $y_{j}$ are regions which are adjacent to an arc $x_{k}$ and the normal direction of $x_{k}$ points away from $y_{i}$ toward $y_{j}$, then

$$
\tilde{\rho}\left(y_{j}\right)=\tilde{\rho}\left(y_{i}\right) * \tilde{\rho}\left(x_{k}\right) .
$$

We call this condition the face coloring condition.

Let $\tau$ be a crossing point and let $x_{i}$ and $x_{k}$ be the arcs around $\tau$ appearing in the crossing relation $x_{j}=x_{i} * x_{k}$ at $\tau$ as before. Let $y$ be the region which is one of the four regions around $\tau$ such that the normal directions of $x_{i}$ and $x_{k}$ are away from the region $y$. Then we give this crossing point $\tau$ an element $W_{\theta}(\tau, \tilde{\rho})$ of $G$ determined by

$$
W_{\theta}(\tau, \tilde{\rho})=\theta\left(\tilde{\rho}(y), \tilde{\rho}\left(x_{i}\right), \tilde{\rho}\left(x_{k}\right)\right)^{\epsilon},
$$

where $\epsilon$ is the sign of the crossing point. Consider the element

$$
\Psi_{\theta}(D)=\sum_{\tilde{\rho}} \prod_{\tau} W_{\theta}(\tau, \tilde{\rho})
$$

of the group ring $\mathbf{Z} G$, where $\tilde{\rho}$ runs all shadow colorings of $D$ by $X$ and $\tau$ runs all crossing points of $D$. Since $\theta$ is a cocycle, the value $\Psi_{\theta}(D)$ is preserved by Reidemeister moves. It is an invariant of the knot $\mathcal{K}$ represented by $D$. We denote this value by $\Psi_{\theta}(\mathcal{K})$. If $\theta$ and $\theta^{\prime}$ are cohomologous, then $\Psi_{\theta}(\mathcal{K})=\Psi_{\theta^{\prime}}(\mathcal{K})$. Thus this invariant depends on the quandle 3-cohomology class of $\theta$. When $\theta$ is a trivial cocycle, the invariant $\Psi_{\theta}(\mathcal{K})$ is equal to the number of the shadow colorings of $D$ by $X$.

Colorings and shadow colorings are defined for oriented knotted surfaces in 4 -space similarly using their diagrams in 3 -space. For a knotted surface $\mathcal{K}$ 
in $\mathbf{R}^{4}$, by modifying it slightly, we may assume that the projection $p: \mathcal{K} \rightarrow$ $\mathbf{R}^{3}$ to the 3 -space is a generic map. The singularity set of the projection consists of double points, triple points and branch points. By removing a small regular neighborhood of the under-curve of the double curve, we have a compact embedded surface in the 3 -space. This is a diagram of the knotted surface $\mathcal{K}$ (see [10] for details). Let $D$ be the diagram and let $E=\left\{x_{1}, \ldots, x_{m}\right\}$ be the set of sheets of the diagram. Using the orientation of $\mathcal{K}$ and the orientation of 3 -space, we give a normal direction (co-orientation) to each sheet. At a double curve of the projection $p(\mathcal{K})$, let $x_{i}, x_{j}$ and $x_{k}$ be the three sheets around the double curve such that $x_{k}$ is the over-sheet and $x_{i}$ and $x_{j}$ are under-sheets and that the normal direction is away from $x_{i}$ toward $x_{j}$. At each double curve, we consider a relation

$$
x_{j}=x_{i} * x_{k}
$$

and call it the crossing relation around the double curve (see Fig. 3). The quandle $Q(D)$ of the diagram is defined to be the quandle generated by $E=$ $\left\{x_{1}, \ldots, x_{m}\right\}$ and the defining relations are the crossing relations around the double curves. This quandle is isomorphic to the quandle $Q(\mathcal{K})$ of the knotted surface $\mathcal{K}$ in the sense of $[12,19]$.

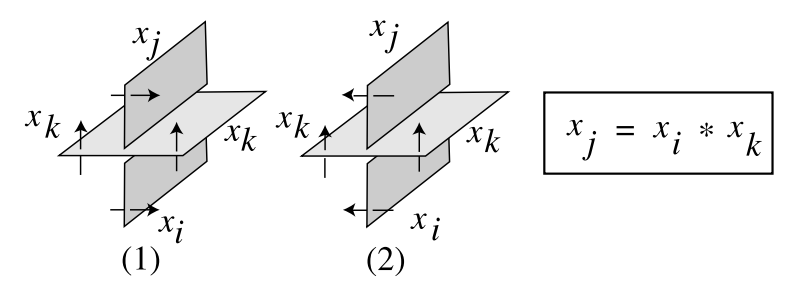

Figure 3

A homomorphism $\rho: Q(D) \rightarrow X$ is called a coloring of the diagram $D$ by $X$. This is equivalent to coloring the sheets by elements of $X$ such that the colors $\rho\left(x_{i}\right), \rho\left(x_{j}\right)$ and $\rho\left(x_{k}\right)$ of the three sheets $x_{i}, x_{j}$ and $x_{k}$ around a double curve satisfies $\rho\left(x_{j}\right)=\rho\left(x_{i}\right) * \rho\left(x_{k}\right)$.

Let $X$ be a finite quandle, let $G$ be an abelian group (written in multiplicative notation), and let $\theta \in C_{\mathrm{Q}}^{3}(X ; G)$ be a quandle 3 -cocycle. For each triple point $\tau$ of the diagram $D$, we consider an element $W_{\theta}(\tau, \rho)$ of $G$ determined by

$$
W_{\theta}(\tau, \rho)=\phi\left(\rho\left(x_{i}\right), \rho\left(x_{j}\right), \rho\left(x_{k}\right)\right)^{\epsilon},
$$

where $x_{i}, x_{j}$ and $x_{k}$ are the sheets around $\tau$ such that $x_{k}$ is the upper-sheet, $x_{j}$ is one of the two middle-sheets from which the normal direction of $x_{k}$ is away, 
and $x_{i}$ is one of the four lower-sheets away from which the normal directions of $x_{k}$ and $x_{j}$ point, and $\epsilon$ is the sign of the triple point $\tau$. Consider the element

$$
\Phi_{\theta}(D)=\sum_{\rho} \prod_{\tau} W_{\phi}(\tau, \rho)
$$

of the group ring $\mathbf{Z} G$, where $\rho$ runs all colorings of $D$ by $X$ and $\tau$ runs all triple points of $D$. Since $\phi$ is a cocycle, the value $\Phi_{\phi}(D)$ is preserved by Roseman moves $([10,26])$. Therefore it is an invariant of the knotted surface $\mathcal{K}$ represented by the diagram. We denote this value by $\Phi_{\phi}(\mathcal{K})$. If $\phi$ and $\phi^{\prime}$ are cohomologous, then $\Phi_{\phi}(\mathcal{K})=\Phi_{\phi^{\prime}}(\mathcal{K})$. Thus this invariant depends on the quandle cohomology class of $\phi$. When $\phi$ is a trivial cocycle, the invariant $\Phi_{\phi}(\mathcal{K})$ is equal to the number of the colorings of $D$ by $X$.

\section{$6 \quad$ Examples}

Let $X$ be the dihedral kei $R_{3}=\{0,1,2\}$ of order three, let $G$ be the cyclic group $\left\langle t \mid t^{3}=1\right\rangle$, and let $\theta \in Z_{\mathrm{Q}}^{3}\left(R_{3} ; \mathbf{Z}_{3}\right)$ be the quandle 3 -cocycle defined by

$$
\theta=\chi_{012} \chi_{021} \chi_{101} \chi_{201} \chi_{202} \chi_{102}
$$

where $\chi_{a b c}(x, y, z)=t$ if $(x, y, z)=(a, b, c)$; otherwise $\chi_{a b c}(x, y, z)=1$. In this situation, we consider the invariant $\Psi_{\theta}$ for classical knots (defined by shadow colorings) and the invariant $\Phi_{\theta}$ for knotted surfaces (defined by colorings). Note that the values $\Psi_{\theta}(\mathcal{K})$ and $\Phi_{\theta}(\mathcal{K})$ are elements of $\mathbf{Z}\left\langle t \mid t^{3}=1\right\rangle$.

Theorem 6.1 (Rourke and Sanderson [27]) The invariant $\Psi_{\theta}$ distinguishes the trefoil $T(2,3)$ and its mirror image $T(2,-3) ; \Psi_{\theta}(T(2,3)) \neq \Psi_{\theta}(T(2,-3))$.

Theorem 6.2 (Carter et al. [9]) A torus knot/link $T(2, n)$ is 3-colorable if and only if $n=3 k$ for some integer $k$. In this case, $\Psi_{\theta}(T(2,3 k))=9+18 t^{k}$.

Theorem 6.3 (Carter et al. [9]) A torus knot/link $T(3, n)$ is 3-colorable if and only if $n=2 k$ for some integer $k$. In this case, if $k$ is not a multiple of 3 , then $\Psi_{\theta}(T(3,2 k))=9+18 t^{k}$; otherwise $\Psi_{\theta}(T(3,2 k))=45$.

There are four knots in the table with less than 8 crossings that are 3 -colorable: $3_{1}, 6_{1}, 7_{4}$, and $7_{7} . \Psi_{\theta}\left(3_{1}\right)=\Psi_{\theta}\left(7_{4}\right)=\Psi_{\theta}\left(7_{7}\right)=9+18 t$ and $\Psi_{\theta}\left(6_{1}\right)=27$, [9]. Further examples are calculated in $[5,9]$. 
Theorem 6.4 (Carter et al. [4], Rourke and Sanderson [27]) Let $\mathcal{K}$ be the 2 -twist spun trefoil and let $-\mathcal{K}$ be the same 2 -knot with the reversed orientation. Then $\Phi_{\theta}(\mathcal{K})=3+6 t^{2}$ and $\Phi_{\theta}(-\mathcal{K})=3+6 t$. Thus the invariant $\Phi_{\theta}$ distinguishes $\mathcal{K}$ and $-\mathcal{K}$.

Carter et al. [5] (Theorem 7.2, Cor. 7.3 and 7.4) and Satoh [28] (Theorem 12) gave formulae on the invariants $\Phi_{\theta}(\mathcal{K})$ for the twist-spun knots. The following theorem is a consequence of the formulae.

Theorem 6.5 (Carter et al. [5], Satoh[28]) Let $\mathcal{K}$ be the $m$-twist spun trefoil. Then

$$
\Phi_{\theta}(\mathcal{K})= \begin{cases}3+6 t^{2} & \text { for } m \equiv 2 \bmod 6 \\ 3+6 t & \text { for } m \equiv 4 \bmod 6 \\ 9 & \text { for } m \equiv 0 \bmod 6 \\ 3 & \text { otherwise }\end{cases}
$$

Remark 6.6 (1) Here we used quandle cohomology of a finite quandle to define the invariants $\Phi$ and $\Psi$. We may use the rack cohomology of a quandle or a rack to define a framed knot. For the purpose of constructing a 3-manifold invariant, one should use the rack homology.

(2) To define the invariants $\Phi$ and $\Psi$, we used the summation over all possible colorings. When we need an invariant of a colored knot, we do not need to take the summation.

Remark 6.7 (1) Shadow coloring of a classical knot diagram is closely related to coloring a knotted surface diagram and its lower decker set. The correspondence is given in [7].

(2) Recall that a shadow coloring of a classical knot diagram is a function $\tilde{\rho}: \tilde{E} \rightarrow X$. The arcs and the regions in $\tilde{E}$ are colored by elements of $X$. However, when we use an $X$-set $Y$ in the sense of Fenn et al. (cf. [13, 14]), we may color the regions with elements of $Y$ and the arcs with elements of $X$. Equivalently, we may introduce and use the cohomologies with twisted coefficients. So there are a lot of variations of the invariants derived from quandle/rack (co)homologies. For example, see [3].

Remark 6.8 Recently, S. Satoh and A. Shima [29] proved that if $\Phi_{\theta}(\mathcal{K})$ is not an integer for a knotted surface $\mathcal{K}$, then the minimum triple point number $t(\mathcal{K})$ of the generic projections of $\mathcal{K}$ is greater than three, where $\Phi_{\theta}$ is the invariant used in this section. As a corollary, they proved that $t(\mathcal{K})=4$ for the 2 -twist spun trefoil $\mathcal{K}$. 


\section{References}

[1] E Brieskorn, Automorphic sets and singularities, Contemporary math. 78 (1988) 45-115

[2] J S Carter, M Elhamdadi, M, A Nikiforou, M Saito, Extensions of quandles and cocycle knot invariants, arXiv:math.GT/0107021

[3] J S Carter, M Elhamdadi, M Saito, Twisted quandle homology theory and cocycle knot invariant, arXiv:math.GT/0108051

[4] J S Carter, D Jelsovsky, S Kamada, L Langford, M Saito, Quandle cohomology and state-sum invariants of knotted curves and surfaces, arXiv:math.GT/9903135 (to appear in Trans. Amer. Math. Soc.)

[5] J S Carter, D Jelsovsky, S Kamada, M Saito, Computations of quandle cocycle invariants of knotted curves and surfaces, Adv. in Math. 157 (2001) 36-94

[6] J S Carter, D Jelsovsky, S Kamada, M Saito, Quandle homology groups, their betti numbers, and virtual knots, J. Pure Appl. Algebra 157 (2001) 135-155

[7] J S Carter, S Kamada, M Saito, Geometric interpretations of quandle homology, J. Knot Theory Ramifications 10 (2001) 345-386

[8] J S Carter, S Kamada, M Saito, Diagrammatic computations for quandles and cocycle knot invariants, arXiv:math.GT/0102092

[9] J S Carter, S Kamada, M Saito, Quandles and cocycle knot invariants, preprint

[10] J Carter, M Saito, Knotted surfaces and their diagrams, American Mathematical Society (1998)

[11] R H Crowell, R H Fox Introduction to Knot Theory. Blaisdell Pub. Co. (1963)

[12] R Fenn, C Rourke, Racks and links in codimension two, J. Knot Theory Ramifications 1 (1992) 343-406

[13] R Fenn, C Rourke, B Sanderson, Trunks and classifying spaces, Appl. Categ. Structures 3 (1995) 321-356

[14] R Fenn, C Rourke, B Sanderson, James bundles and applications, preprint

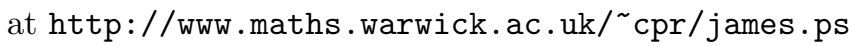

[15] J Flower, Cyclic Bordism and Rack Spaces, Ph.D. Dissertation, Warwick (1995)

[16] RH Fox, A quick trip through knot theory, in Topology of 3-Manifolds, Prentice-Hall (1962) 120-167

[17] M T Greene, Some Results in Geometric Topology and Geometry, Ph.D. Dissertation, Warwick (1997)

[18] A Inoue, Quandle homomorphisms of knot quandles to Alexander quandles, J. Knot Theory Ramifications 10 (2001) 813-822

[19] D Joyce, A classifying invariant of knots, the knot quandle, J. Pure Appl. Alg. 23 (1982) 37-65 
[20] L H Kauffman, Knots and Physics, World Scientific (1991)

[21] R A Litherland, S Nelson, The Betti numbers of some finite racks, arXiv: math.GT/0106165

[22] P Lopes, Quandles at finite temperatures I, arXiv:math.QA/0105099

[23] S Matveev, Distributive groupoids in knot theory, (Russian) Mat. Sb. (N.S.) 119(161) (1982) 78-88, 160

[24] T Mochizuki, Some calculations of cohomology groups of finite Alexander quandles, preprint

[25] J H Przytycki, 3-coloring and other elementary invariants of knots, in Banach Center Publications 42 (1998) Knot theory, 275-295

[26] D Roseman, Reidemeister-type moves for surfaces in four dimensional space, in Banach Center Publications 42 (1998) Knot theory, 347-380

[27] C Rourke, B Sanderson, There are two 2-twist-spun trefoils, arXiv: math.GT/0006062

[28] S Satoh, Surface diagrams of twist-spun 2-knot, preprint, to appear in the proceedings of "Knots 2000", (Korea, 2000)

[29] S Satoh, A Shima, The 2-twist-spun trefoil has the triple point number four, preprint

[30] D S Silver, S G Williams, Generalized n-colorings of links, in Banach Center Publications 42 (1998) Knot theory, 381-394

[31] M Takasaki, Abstraction of symmetric transformations, (in Japanese) Tohoku Math. J. 49 (1942/43) 145-207

[32] S Winker, Quandles, knots invariants and the $n$-fold branched cover, Ph.D. Thesis, University of Illinois at Chicago (1984)

Dept. of Math., Hiroshima University, Hiroshima 739-8526, JAPAN

Email: kamada@math.sci.hiroshima-u.ac.jp

URL: http://www.math.sci.hiroshima-u.ac.jp/ kamada/index.html 